\title{
Experimental evidence and molecular modeling of the interaction between hRSV-NS1 and quercetin
}

\author{
Deriane Elias Gomes a,b ${ }^{\mathrm{a}}$, Icaro Putinhon Caruso ${ }^{\mathrm{a}, \mathrm{b}}$, Gabriela Campos de Araujo ${ }^{\mathrm{a}, \mathrm{b}}$, \\ Isabella Otenio de Lourenço ${ }^{a, b}$, Fernando Alves de Melo ${ }^{a, b}$, Marinônio Lopes Cornélio ${ }^{a, b}$, \\ Marcelo Andrés Fossey ${ }^{\mathrm{a}, \mathrm{b}}$, Fátima Pereira de Souza ${ }^{\mathrm{a}, \mathrm{b}, *}$ \\ a Departamento de Física-Instituto de Biociências, Letras e Ciências Exatas (IBILCE) Universidade Estadual Paulista "Júlio de Mesquita Filho" \\ (UNESP)-Campus de São José do Rio Preto-SP. Rua Cristóvão Colombo, 2265, Jardim Nazareth, Cep: 15054-000 São José do Rio Preto, SP, Brazil \\ ${ }^{\mathrm{b}}$ Centro Multiusuário de Inovação Biomolecular (CMIB)-Instituto de Biociências, Letras e Ciências Exatas (IBILCE) Universidade Estadual Paulista "Júlio de \\ Mesquita Filho" (UNESP)_Campus de São José do Rio Preto-SP. Rua Cristóvão Colombo, 2265, Jardim Nazareth, Cep: 15054-000 São José do Rio Preto, SP, \\ Brazil
}

\section{A R T I C L E I N F O}

\section{Article history:}

Received 14 November 2014

Received in revised form

29 November 2015

Accepted 14 December 2015

Available online 21 December 2015

\section{Keywords:}

Non structural protein 1

Flavonoids

Quercetin

Fluorescence spectroscopy

Molecular modeling

Human syncytial respiratory virus

\begin{abstract}
A B S T R A C T
Human Respiratory Syncytial Virus is one of the major causes of acute respiratory infections in children, causing bronchiolitis and pneumonia. Non-Structural Protein 1 (NS1) is involved in immune system evasion, a process that contributes to the success of hRSV replication. This protein can act by inhibiting or neutralizing several steps of interferon pathway, as well as by silencing the hRSV ribonucleoproteic complex. There is evidence that quercetin can reduce the infection and/or replication of several viruses, including RSV. The aims of this study include the expression and purification of the NS1 protein besides experimental and computational assays of the NS1-quercetin interaction. CD analysis showed that NS1 secondary structure composition is 30\% alpha-helix, $21 \%$ beta-sheet, $23 \%$ turn and $26 \%$ random coils. The melting temperature obtained through DSC analysis was around $56^{\circ} \mathrm{C}$. FRET analysis showed a distance of approximately $19 \AA$ between the NS1 and quercetin. Fluorescence titration results showed that the dissociation constant of the NS1-quercetin interaction was around $10^{-6} \mathrm{M}$. In thermodynamic analysis, the enthalpy and entropy balanced forces indicated that the NS1-quercetin interaction presented both hydrophobic and electrostatic contributions. The computational results from the molecular modeling for NS1 structure and molecular docking regarding its interaction with quercetin corroborate the experimental data.
\end{abstract}

(C) 2015 Elsevier B.V. All rights reserved.

\section{Introduction}

The participation of Human Respiratory Syncytial Virus (hRSV) in respiratory diseases during childhood can be life-threatening, with severe consequences like pneumonia and bronchiolitis. The major strategy that contributes to the success of hRSV replication is its immune system evasion efficacy, a process provided by NonStructural Proteins (NS1 and NS2). NS1 is a small protein that has 139 amino acids [1] with $15 \mathrm{kDa}$. Extensive studies have shown that

\footnotetext{
* Corresponding author.

E-mail addresses: derianegomes1@gmail.com (D.E. Gomes), ykrocaruso@hotmail.com (Î.P.Caruso),bicamposaraujo@hotmail.com (G.C.d. Araujo), isabella_o.lourenco@hotmail.com (I.O.d. Lourenço), fernanmello@gmail.com (F.A.d. Melo), mario@ibilce.com.br (M.L. Cornélio), marcelo@ibilce.unesp.br (M.A. Fossey), fatyssouza@gmail.com, fatyssouza@yahoo.com.br (F.P.d. Souza).
}

NS1 plays an important role in the modulation of the host response to infection, antagonizing the interferon-mediated antiviral state [2].

It has been reported that NS1 (1) co-localizes with the mitochondrial signaling protein (MAVS), inhibiting MAVS-RIG-I interaction and decreasing protein levels of members of the IFN induction pathway, such as TRAF3 and IKKe [3,4]; (2) interacts with microtubule-associated protein 1B (MAP1B), which may be important for surface recognition of these proteins with other host structures [5]; and (3) interacts with M protein and the $C$-terminus of $P$ protein [6].

Flavonoids have gained world interest due to their nutraceutical and therapeutic importance, exhibiting several biological activities such as antioxidant, anti-inflammatory, cardioprotective, antibacterial, antitumor, hepatoprotective, and antiviral activities [7]. Thus, because quercetin is the most studied flavonoid and it has antiviral properties [8-11], we decided to investigate how 
quercetin interacts with NS1, and affects its immune-system evasion efficiency.

Flavonoids are useful model molecules for spectroscopy studies due to their interactions with relevant target proteins and some spectroscopic profiles of quercetin such as (1) the excited-state intramolecular proton transfer, (2) dual fluorescence behavior and (3) the high intensity of the fluorescence signal in low polar solvents [12]. Biophysical binding properties have been explored in some detail using human serum albumin (HSA) and quercetin as binding partners [12]. Furthermore, the low toxicity and pharmacokinetics of quercetin are described in the literature [13], with reduced therapeutic risk. In the present study, a combination of experimental procedures with molecular docking has elucidated several aspects of the interaction between NS1 and quercetin.

\section{Materials and methods}

\subsection{NS1 expression and purification}

Human Syncytial Respiratory Virus (type A) NS1 codon optimization and pJexpress401-NS1 construction were developed by DNA2.0 [14] BL-21(DE3) cells containing the plasmid pJexpress401-NS1 grown in $1.5 \mathrm{~L}$ of $2 \mathrm{YT}$ medium containing $25 \mu \mathrm{g} / \mathrm{mL}$ kanamycin and $34 \mu \mathrm{g} / \mathrm{mL}$ chloramphenicol with constant shaking at $37^{\circ} \mathrm{C}$ until they reached the optical density of 0.8 . Induction was performed with $0.5 \mathrm{mM}$ IPTG (final concentration) for three hours at the same temperature. Cultures were harvested by centrifugation at $4{ }^{\circ} \mathrm{C}, 7000 \mathrm{rpm}$, for $30 \mathrm{~min}$ and the pellet cells were stored at $-80^{\circ} \mathrm{C}$ until use.

Cells were suspended in $40 \mathrm{~mL}$ lysis buffer (50 mM Tris- $\mathrm{HCl}$; $100 \mathrm{mM} \mathrm{NaCl} ; 5 \mathrm{mM} \beta \mathrm{Me}, \mathrm{pH}$ 8.0) containing Animal Component Free Protease Inhibitor Cocktail (Sigma ${ }^{\circledR}$, I3911-1BO) and were disrupted by sonication. The lysed cells were centrifuged at $4{ }^{\circ} \mathrm{C}$, $7000 \mathrm{rpm}$, for $30 \mathrm{~min}$ and the supernatant was loaded at a constant flow rate of $0.5 \mathrm{~mL} / \mathrm{min}$, in a Nickel Resin His60 Ni Superflow $\left(\right.$ Clontech ${ }^{\circledR}$ ), which was previously equilibrated with purification buffer (PB - $50 \mathrm{mM}$ TRIS, $100 \mathrm{mM} \mathrm{NaCl}, 1 \mathrm{mM} \beta \mathrm{Me}, \mathrm{pH} 8.0$ ), coupled to an AKTA Purifier $\left(\mathrm{GE}^{\circledR}\right)$. After that, the column was washed with $\mathrm{PB}$ to remove non-binding proteins from lysed cells and followed by $\mathrm{PB}$ containing $40 \mathrm{mM}$ imidazole to remove non-specific bound contaminants. NS1 elution was performed using PB containing $200 \mathrm{mM}$ imidazole and the fractions collected were analyzed by SDS-PAGE. Pure fractions were desalted and stored at $4{ }^{\circ} \mathrm{C}$.

NS1 desalting was performed on a PD-10 Desalting Column (GE Healthcare $\left.{ }^{\circledR}\right)$ equilibrated with buffer $\left(0.05 \mathrm{mM} \mathrm{NaH}_{2} \mathrm{PO}_{4}, 300 \mathrm{mM}\right.$ $\mathrm{NaCl}$ at $\mathrm{pH} 7.4$ ) and kept on ice after that for up to $24 \mathrm{~h}$ before the interaction assays. Quercetin stock solution was prepared in $90 \%$ ethanol v/v and kept on ice after that for up to $24 \mathrm{~h}$ before the interaction assays.

\subsection{UV-Vis absorbance spectroscopy}

UV-Vis measurements were performed on a Cary-3E spectrophotometer (Varian, Palo Alto, CA) equipped with a quartz cell of $1.0 \mathrm{~cm}$ path length, under the following conditions: scanning speed of $600 \mathrm{~nm} / \mathrm{min}, 1.0 \mathrm{~nm}$ of interval and spectral bandwidth of $2.0 \mathrm{~nm}$. The spectra were performed at room temperature. The stock concentrations were obtained using the following extinction coefficients: $\varepsilon_{375 \mathrm{~nm}}=21.880 \mathrm{M}^{-1} \mathrm{~cm}^{-1}$ for quercetin (Sigma-Aldrich ${ }^{\circledR}$, Q4951) (at $375 \mathrm{~nm}$ ) and $\varepsilon_{280 \mathrm{~nm}}=10.220^{-1} \mathrm{M}^{-1} \mathrm{~cm}^{-1}$ (at $280 \mathrm{~nm}$ ) for NS1 (Expasy-ProtParam [15]), obtained the final concentrations of $17.6 \mu \mathrm{M}$ for NS1 and $7.1 \mathrm{mM}$ for quercetin.

\subsection{Circular dichroism spectroscopy $(C D)$}

Far-UV CD measurements were carried out on a Jasco J-810 spectropolarimeter (Jasco, USA) equipped with a quartz cell of $0.01 \mathrm{~cm}$ path length. The CD spectrum range $185-260 \mathrm{~nm}$ was recorded at a scan rate of $20 \mathrm{~nm} / \mathrm{min}$, a response time of $2.0 \mathrm{~s}$, and spectral bandwidth of $1.0 \mathrm{~nm}$. For the NS1 spectrum, six accumulations were performed. The measurement was performed at room temperature (with NS1 concentration of $17.6 \mu \mathrm{M}$ ). The CD spectrum was taken as millidegrees $(\theta)$ and then expressed in terms of mean residue ellipticity (MRE or $[\Theta]$ ) in $\operatorname{deg} \mathrm{cm}^{2} \mathrm{dmol}^{-1}$ using the following equation:

$[\Theta]=\frac{\theta(\mathrm{mdeg})}{(10[P] \ln )}$

where $[P]$ is the molar concentration of the protein, $n$ is the quantity of amino acid residue, and $l$ is the path length $(\mathrm{cm})$. The secondary structures of the protein NS1 were calculated with CONTINLL software from the CDPro package, using the reference set of proteins SMP37 [16].

\subsection{Differential scanning calorimetry}

Differential scanning calorimetry (DSC) measurements were recorded on a N-DSC III (TA Instruments, USA) in the temperature range of $20-80 \mathrm{C}$ with a heating and cooling scan rate of $1 \mathrm{C} / \mathrm{min}$. NS1 was diluted in phosphate buffer $\left(50 \mathrm{mM} \mathrm{NaH}_{2} \mathrm{PO}_{4}, 50 \mathrm{mM}\right.$ $\mathrm{NaCl}, \mathrm{pH} 8.0$ ) to a final concentration of $4.4 \mu \mathrm{M}$. Both calorimeter cells were loaded with the buffer solution, equilibrated at $20^{\circ} \mathrm{C}$ for $10 \mathrm{~min}$ and scanned repeatedly as described above until the baseline was reproducible. Afterwards the sample cell was loaded with NS1 solution and scanned in the same way. Baseline correction was conducted by subtracting the 'buffer vs., buffer' scan from the corresponding 'protein vs., buffer' scan.

\subsection{Fluorescence spectroscopy}

The fluorescence spectroscopy measurements were performed using a Cary Eclipse Fluorescence Spectrophotometer (Varian, Palo Alto, CA) equipped with a quartz cell of $1.0 \mathrm{~cm}$ path length and a Peltier Single Cell Holder System. Both excitation and emission bandwidths were set at $4 \mathrm{~nm}$. The excitation wavelength at $280 \mathrm{~nm}$ and emission spectrum were collected in the range $290-500 \mathrm{~nm}$, which was corrected for the background fluorescence of the buffer and for the inner filter effect [17]. The fluorescence quenching experiments were carried out at the temperatures of 25 and $37^{\circ} \mathrm{C}$. The titrations were performed by adding small aliquots $(\leq 10 \mu \mathrm{L})$ from quercetin stock solution to NS1 solution $(2.0 \mathrm{~mL})$ at a constant concentration of $1.1 \mu \mathrm{M}$. The final concentration of quercetin achieved in the protein solution was $25.6 \mu \mathrm{M}$. In every experiments, the final volume of ethanol in the buffer was $2.7 \%$.

\subsection{Molecular modeling of NS1}

Since the NS1 model had not been proposed previously, protein modeling techniques were used to obtain a structural model. Due to low homology with other proteins with known atomic coordinates, the NS1 modeling method applied was the Rosetta Scoring function and fragment insertion methodologies were developed for ab initio structure prediction [18]. First, a customized library of fragments for each three and nine residue in the protein sequence was selected from a database of known protein structure. Then these fragments were assembled using Monte Carlo simulated annealing search strategy in which fragments are randomly inserted into the protein sequence by replacing the backbone torsion angles in the protein chain with those in the fragment. For NS1 protein (145 aa), 56,475 structures were generated. Two structures with the lowest 

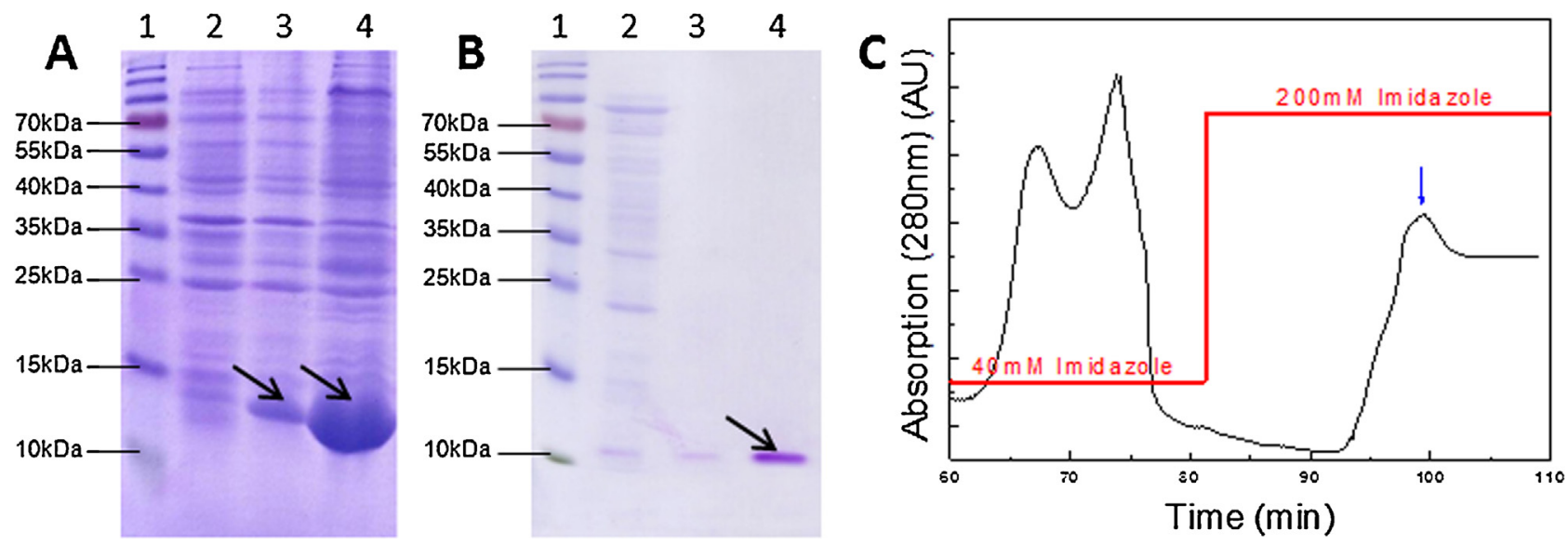

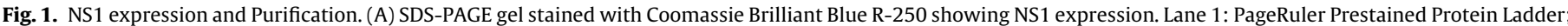

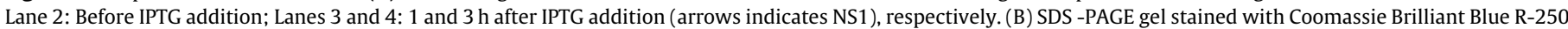

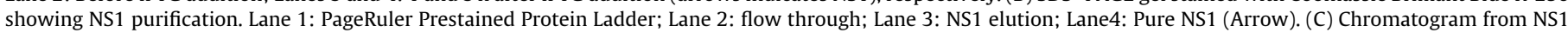

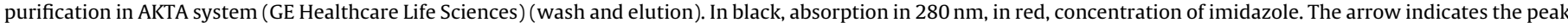
of pure protein.

scores (as assessed by the number of the other 56,474 structures within 3 Årmsd [19]) were visually inspected. The quality of the model was validated using the PROQ [20] and the PROCHECK [21] servers. The second model was showed to be like the first model but with better estimated accuracy and agreement with experimental data. The Dali server was used to search for native-like substructures [22].

\subsection{Molecular dynamics simulation of NS1 in solution}

Molecular dynamics simulations were performed using a GROMACS molecular dynamics package (version 4.5.5) [23,24] to confirm the stability of the NS1 protein model in water containing $150 \mathrm{mM} \mathrm{NaCl}$. We used the GROMOS53A6 force field [25] to model the NS1 protein and SPC as a water model [26]. Temperature was kept constant at $310 \mathrm{~K}$ by means of a V-rescale [27] thermostat with coupling constant tau $=0.1$. Pressure was held constant at $1 \mathrm{~atm}$ by a Berendsen barostat [28] with coupling constant tau $=1$. Integration of Newton's equation of motion was performed by the leap-frog algorithm with a time step of $2 \mathrm{fs}$. Long-range electrostatic interaction was treated using fast particle-mesh Ewald electrostatics (PME) [29]. A cutoff of $1.25 \mathrm{~nm}$ was implemented for the Lennard-Jones and the direct space part of the Ewald sum for Coulombic interactions. The Fourier space part of the Ewald splitting was computed by using the particle-mesh Ewald method [30], with a grid length of $0.15 \mathrm{~nm}$. The overall electric charge of the system was compensated by adding $\mathrm{Cl}^{-}$or $\mathrm{Na}^{+}$ions, respectively.

The simulation starting from the NS1 structure constructed by ROSETTA, was equilibrated by 100 ps of MD with position restraints on the protein to allow for the relaxation of the solvent molecules. This first equilibration run was followed by another 50-ps run without position restraints on the protein. The production run at constant temperature and pressure conditions, after equilibration, was $10 \mathrm{~ns}$ long. Stability of the NS1 model molecule was determined by means of the root mean square deviation (RMSD) using tools.

\subsection{Docking calculation of the NS1-quercetin complex}

The g_cluster tool from the GROMACS package [31] was used to select the most likely NS1 model. The molecular structure of the quercetin was obtained from PRODRG [32]. AutoDockTools (ADT) software [33] of the MGL Tools 1.5.4 program was used to prepare the NS1 and quercetin. The protonation state of histidine side chains was set up according to the PropKa server [34], also using ADT software. The grid maps were generated with $0.375 \AA$ spacing and dimensions of $126 \times 126 \times 126$ points by the AutoGrid 4.2 program [35]; these maps were centered in the protein. The AutoDock 4.2 program [35] was used to study the binding site between quercetin and NS1 by applying the Lamarckian Genetic Algorithm (LGA) for minimization using 50 million energy evaluations, a population size of 300 and root-square-mean deviation (RMSD) tolerance for cluster analysis of $2.0 \AA ̊$. For each docking simulation, 100 different conformers were generated. The ADT software was used to visualize the docked conformations and to identify the interactions between amino acid residues of NS1 and quercetin. Chimera software [36] was used to generate the representation figures of the modeling and docking calculation. The map of the interactions involved in the binding site was calculated using LigPlot program [37].

\section{Results and discussion}

\subsection{NS1 expression, purification and characterization}

\subsubsection{NS1 expression and purification}

Fig. 1AA shows the results for NS1 expression in which the sample collected before induction with IPTG, and at 1 hour and $3 \mathrm{~h}$ of growth after induction with IPTG can be seen in the second, third and fourth lanes, respectively. The most promising condition for protein expression was reached by growing cells for $3 \mathrm{~h}$ after induction by using $0.5 \mathrm{mM}$ IPTG, at $37^{\circ} \mathrm{C}$. The best condition for NS1 purification was determined washing two column volumes with purification buffer containing $40 \mathrm{mM}$ of imidazole before eluting it against the purification buffer containing $200 \mathrm{mM}$ imidazole, see Fig. 1C. The results can be seen in Fig. 1B in lanes 3 and 4, respectively.

\subsubsection{Secondary structure analysis}

The structural characteristics of NS1 in solution were studied using the CD technique. The far UV-CD spectrum of the NS1 presented characteristics of a structured protein from its minimum at $209 \mathrm{~nm}$ and positive ellipticity at $190 \mathrm{~nm}$ (Fig. 2). The CD spectrum of the NS1 presented the same spectral profile described in the literature [38]. The secondary structure analysis was performed using CONTINLL software [16] and indicating: 30\% of $\alpha$-helix, 21\% of $\beta$-sheet, $23 \%$ of turn, and $26 \%$ of random coil. 


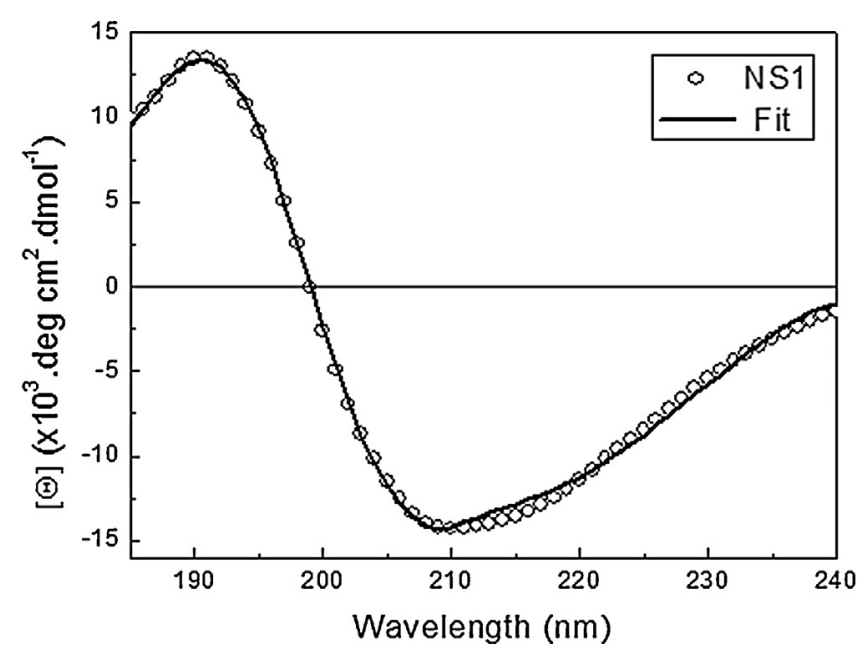

Fig. 2. Far UV-CD spectrum of NS1 $(17.6 \mu \mathrm{M})$ which is represented by unfilled circles. The black line represents the best result for curve fit performed by CONTINLL program.

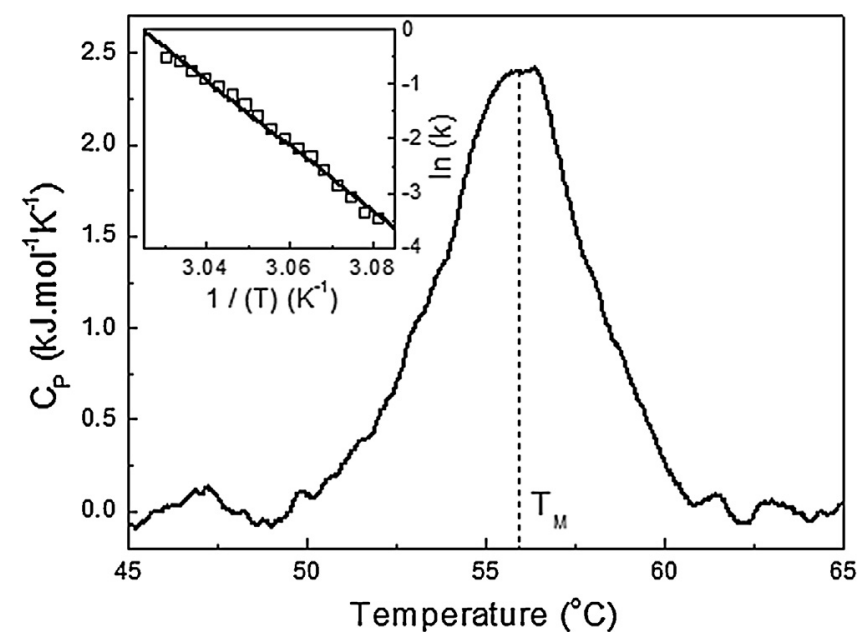

Fig. 3. DSC thermogram of the unfolding process of NS1. The apparent excess heat capacity curve was obtained for $\mathrm{NS} 1(4.4 \mu \mathrm{M})$ in phosphate buffer $\left(50 \mathrm{mM} \mathrm{NaH}_{2} \mathrm{PO}_{4}\right.$, $50 \mathrm{mM} \mathrm{NaCl}, \mathrm{pH} 8.0$ ) at a scan rate of $1^{\circ} \mathrm{C} / \mathrm{min}$. The dotted lines (- - ) indicates the melting temperature $\left(T_{M}\right)$ of the protein. The insert corresponds to the Arrhenius plot of the unfolding process of NS1.

\subsubsection{Thermal characterization}

The thermal stability of NS1 was investigated using the DSC technique. Fig. 3 shows the DSC thermogram of the unfolding process (heating scan) of the protein. It can be seen from the thermogram that NS1 presents a melting temperature $\left(\mathrm{T}_{M}\right)$ at approximately $56^{\circ} \mathrm{C}$, with the endothermic transition starting at about $50^{\circ} \mathrm{C}$ and ending at $61^{\circ} \mathrm{C}$, This result corroborates the thermal denaturation study of NS1 by using CD spectroscopy in the literature, in which a melting temperature at $55^{\circ} \mathrm{C}$ was obtained [38]. The cooling scan after the denaturation presented no transitions in the DSC thermogram of NS1, indicating that its thermal unfolding is irreversible as is also shown in the literature [38]. The calorimetric enthalpy change $\left(\Delta \mathrm{H}_{\text {cal }}\right)$ was determined to be $12 \mathrm{~kJ} \mathrm{~mol}^{-1}$ from the total integral area under the thermogram peak. The rate constant $(k)$ of the unfolding process at a given temperature can be obtained by using:

$k=\frac{v C_{P}}{\left(\Delta H_{\mathrm{cal}}-\mathrm{Q}\right)}$

where $v\left(1.0^{\circ} \mathrm{Cmin}^{-1}\right)$ stands for the scan rate, $C_{P}$ for the excess heat capacity, and $Q$ for the heat evolved at a given temperature.
A value of $499 \mathrm{~kJ} \mathrm{~mol}^{-1}$ for the activation energy can be calculated from the Arrhenius plot in the insert of Fig. 3 [39].

\subsection{Characterization of the interaction between NS1 and quercetin}

\subsubsection{Fluorescence spectra and Förster resonance energy transfer}

Fig. 4aa shows the fluorescence emission spectra of the NS1 in the absence and presence of quercetin. The fluorescence spectrum of the NS1 excited at $280 \mathrm{~nm}$ presented an emission profile of its unique tryptophan residue (Trp) with an intensity maximum at $326 \mathrm{~nm}$ [38]. With the increment in the quercetin concentration in the NS1 solution, the fluorescence intensity of the protein at $326 \mathrm{~nm}$ was quenched with a concomitant increase in the intensity at $490 \mathrm{~nm}$ (see Fig. 4b), corresponding to quercetin emission [40-42]. Under the same condition, blank quercetin in the absence of NS1 does not show any detectable fluorescence [41]. Consequently, the fluorescence increment observed at $490 \mathrm{~nm}$ is due to the interaction between quercetin and NS1 and, subsequently, the resonance energy transfer of Trp residue to the flavonoid. In the literature, there are similar results demonstrating the resonance energy transfer between serum albumin and quercetin [41,42]. The energy transfer parameters and the average distance between the Trp residue in NS1 and quercetin was determined by applying Förster's theory of resonance energy transfer (FRET) [43].

FRET is a photophysical process that involves the transfer of the excitation energy of an electronically excited donor to an acceptor molecule via non-radiative routes. According to Förster's non-radiative energy transfer theory, it occurs under the following conditions: when the emission spectrum of the donor and the absorbance spectrum of the acceptor have a partial overlap; when orientation of the transition dipole of the donor and acceptor occurs; and when the distance between them is less than $8.0 \mathrm{~nm}$. According to the theory, the distance $r$ reflects the approximation between donor (NS1-Trp) and acceptor (Quercetin) which can be calculated by the equation:

$E=1-\left(\frac{F}{F_{0}}\right)=\frac{R_{0}^{6}}{\left(R_{0}^{6}+r^{6}\right)}$

where $E$ is the efficiency of energy transfer, $F_{0}$ and $F$ are the fluorescence intensity in the absence and presence of the acceptor (quercetin), respectively, and $R_{0}$ is the critical distance when the transfer efficiency is 50\%:

$R_{0}^{6}=8.79 \times 10^{-25} K^{2} n^{-4} \phi J$

In Eq. (4), $K^{2}$ is the orientation factor related to the geometry of the donor (NS1-Trp) and acceptor (quercetin) and has been adopted to be $2 / 3$ [44], which randomizes by rotational diffusion prior to energy transfer; $n$ is the average refracted index of the medium in the wavelength range in which spectral overlap is significant and is taken as $1.36 ; \phi$ is the fluorescence quantum yield of the donor which was calculated taking the quantum yield of serum albumin a reference $[45,46]$; and $J$ is the effect of the spectral overlap between the emission spectrum of the donor and the absorption spectrum of the acceptor (see Fig. 5), which may be calculated by the following equation:

$J=\frac{\int_{0}^{\infty} F(\lambda) \varepsilon(\lambda) \lambda^{4} d \lambda}{\int_{0}^{\infty} F(\lambda) d \lambda}$ 

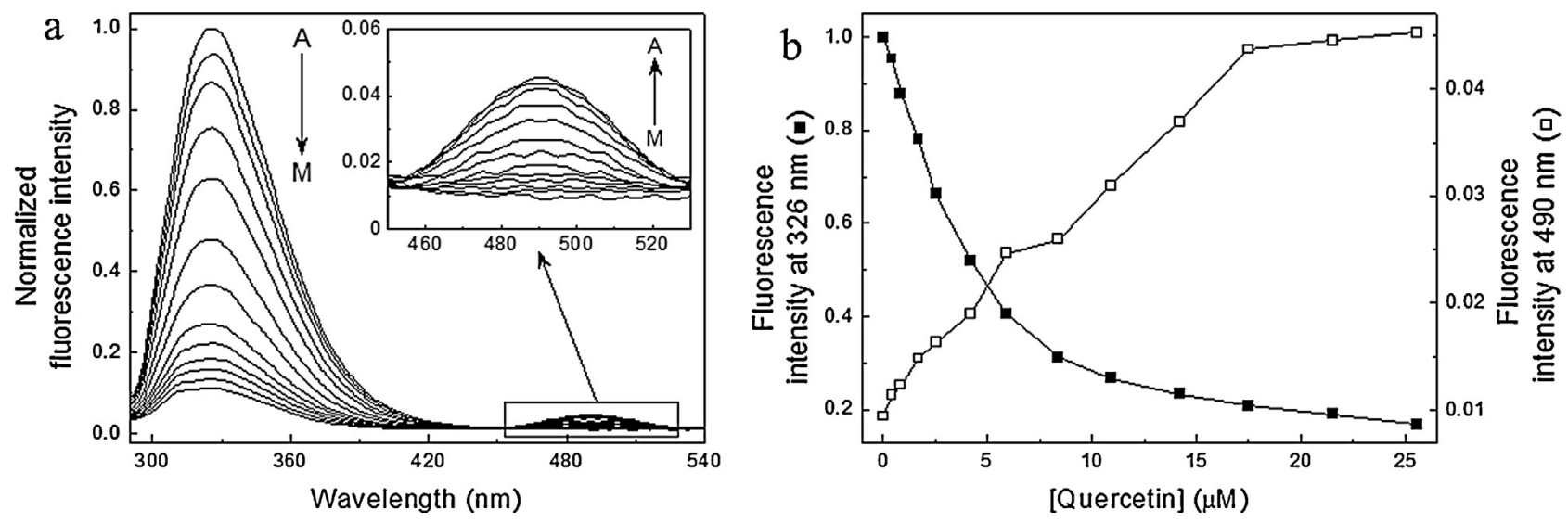

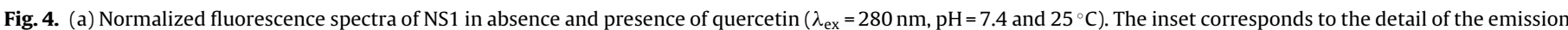

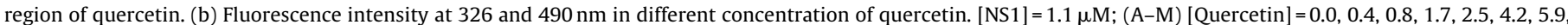
$8.4,10.9,14.2,17.5,21.5$ and $25.6 \mu \mathrm{M}$.

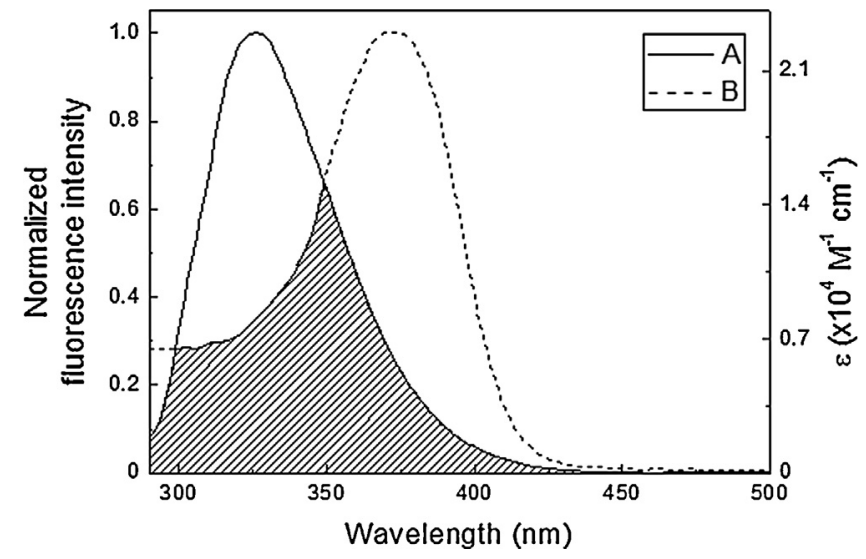

Fig. 5. Spectral overlaps of the NS1 fluorescence (A) with quercetin absorption (B).

where $F(\lambda)$ is the corrected fluorescence intensity of the donor in the wavelength range from $\lambda$ to $\lambda+\Delta \lambda$ and $\varepsilon(\lambda)$ is the extinction coefficient of the acceptor at $\lambda$.

In order to obtain the average distance between NS1-Trp and quercetin, the $F / F_{0}$ value in Eq. (3) was calculated by extrapolating to zero at the linear of plot $F / F_{0}$ vs., $1 /$ [Quercetin], which gave a value of 0.107 (Supplementary material, Fig. S1). Thus, the efficiency of energy transfer $(E)$ were determined at the saturation point and its value was 0.893 . According to Eqs. (3)-(5), the values of $J=3.776 \times 10^{-15} \mathrm{~cm}^{3} \mathrm{~L} \mathrm{~mol}^{-1}, R_{0}=2.715 \mathrm{~nm}$ and $r=1.906 \mathrm{~nm}$ (or $19.06 \AA$ ) was calculated. The average distance between donor and acceptor fluorophore is in the range $0.5 R_{0}<r<1.5 R_{0}$ [43-45], which indicates that energy transfer from NS1-Trp and Quercetin occurs with high probability.

\subsubsection{Dissociation constant of the NS1-QCT complex}

The dissociation constants $\left(K_{d}\right)$ for the interaction between NS1 and quercetin were determined by fluorescence quenching change analysis (Fig. 6). The fluorescence titration data at 25 and $37^{\circ} \mathrm{C}$ were fitted using the Eq. (6), which describes ligand binding to a single protein site (1:1 complex) [47]:

$\frac{F_{0}-F}{F_{0}-F_{S}}=\frac{\left(\left[P_{T}\right]+\left[L_{T}\right]+K_{d}\right)-\sqrt{\left(\left[P_{T}\right]+\left[L_{T}\right]+K_{d}\right)^{2}-4\left[P_{T}\right]\left[L_{T}\right]}}{2\left[P_{T}\right]}$

where $F_{0}$ and $F$ are the steady-state fluorescence intensities in the absence and presence of quercetin, respectively. $F_{S}$ is the fluorescence intensity of the fully complexed protein (saturated NS1 with

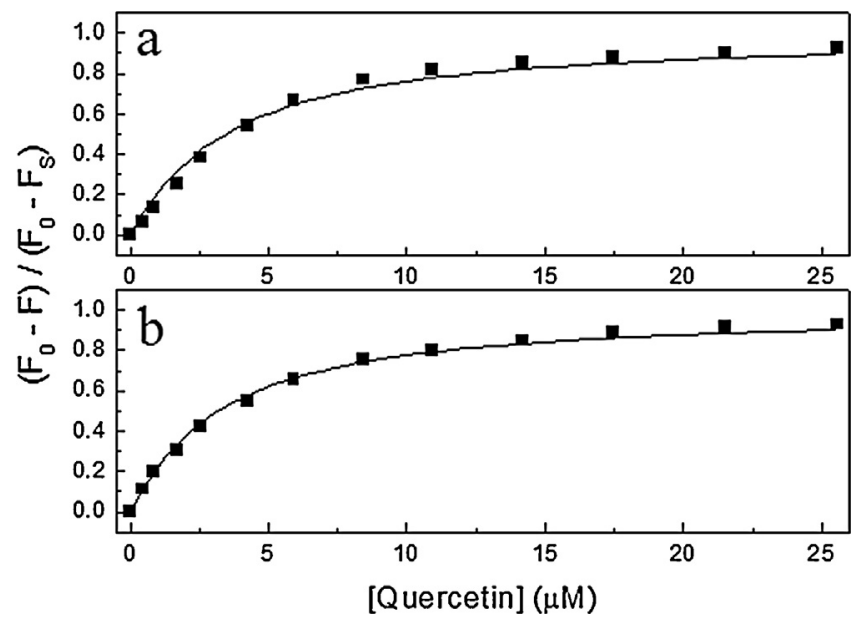

Fig. 6. Fluorescence quenching change of NS1 $\left(\lambda_{\mathrm{em}}=326 \mathrm{~nm}\right)$ in function of quercetin concentration at (a) 25 and (b) $37^{\circ} \mathrm{C}$. The lines correspond to the adjustments of the experimental data made using Eq. (6). All the correlation coefficient are $\geq 0.99$.

quercetin), which was obtained by extrapolation at the plot of Fig. S1 (Supplementary material), $\left[P_{T}\right]$ is the total concentration of NS1 and $\left[L_{T}\right]$ is the total concentration of quercetin.

$K_{d}$ values for the NS1-quercetin interaction at 25 and $37^{\circ} \mathrm{C}$ are shown in Table 1. It can be observed in Table 1 that, notwithstanding the fact that $K_{d}$ values are similar at the two temperatures, a slight decrease of dissociation constant value occurs with the increase from 25 to $37^{\circ} \mathrm{C}$.

\subsubsection{Thermodynamic analysis}

The protein-ligand interaction may involve the formation of hydrogen bonds, Van der Waals, electrostatic forces, and hydrophobic interactions. In order to elucidate the interactions between NS1 and quercetin, the enthalpy change $(\Delta H)$ was calculated from the van't Hoff equation:

$\ln \left(\frac{K_{d 1}}{K_{d 2}}\right)=\left(\frac{-\Delta \mathrm{H}}{\mathrm{R}}\right)\left(1 / \frac{T_{2-1}}{T_{1}}\right)$

where $K_{d 1}$ and $K_{d 2}$ are dissociation constants at the absolute temperatures $T_{1}$ and $T_{2}$, which were $298 \mathrm{~K}\left(25^{\circ} \mathrm{C}\right)$ and $310 \mathrm{~K}\left(37^{\circ} \mathrm{C}\right)$, respectively. $R$ corresponds to the universal gas constant. The Gibbs 
Table 1

Dissociation constants $\left(K_{d}\right)$ and thermodynamic parameters for NS1-quercetin interaction.

\begin{tabular}{|c|c|c|c|c|c|}
\hline$T\left({ }^{\circ} \mathrm{C}\right)$ & $K_{\mathrm{d}(\mu \mathrm{M})}$ & $\begin{array}{l}\Delta H \\
\left(\mathrm{~kJ} \mathrm{~mol}{ }^{-1}\right)\end{array}$ & $\begin{array}{l}-T \Delta S \\
\left(\mathrm{~kJ} \mathrm{~mol}^{-1}\right)\end{array}$ & $\begin{array}{l}\Delta G \\
\left(\mathrm{~kJ} \mathrm{~mol}^{-1}\right)\end{array}$ & $\begin{array}{l}\Delta S \\
\left(\mathrm{~kJ} \mathrm{~mol}{ }^{-1} \mathrm{~K}^{-1}\right)\end{array}$ \\
\hline 25 & 2.86 & 5.1 & -36.7 & -31.6 & 0.123 \\
\hline 37 & 2.64 & & -38.2 & -33.1 & \\
\hline
\end{tabular}

$T$ : temperature; $\Delta H$ : enthalpy change; $\Delta G$ : Gibbs free energy change; $\Delta S$ : entropy change.

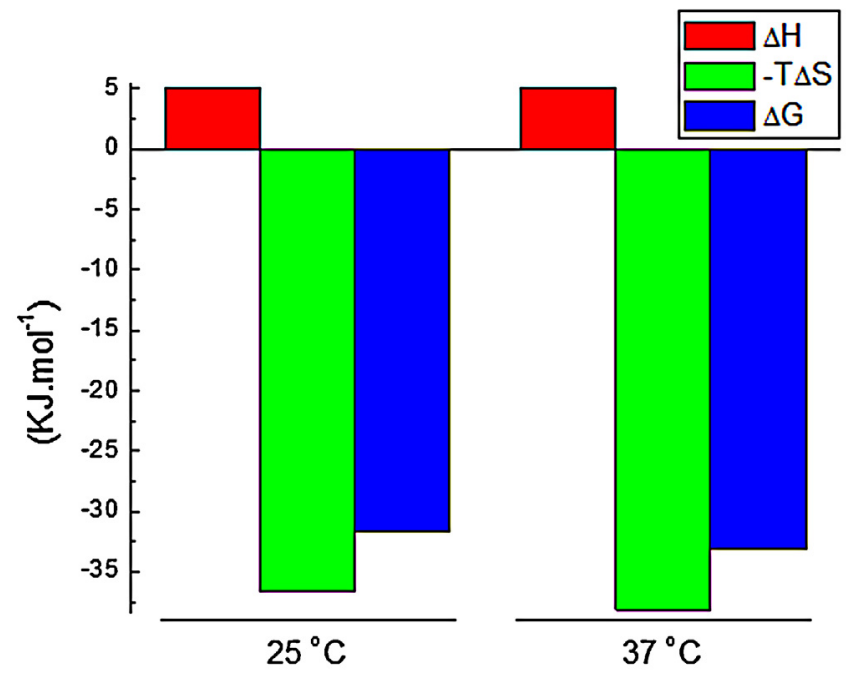

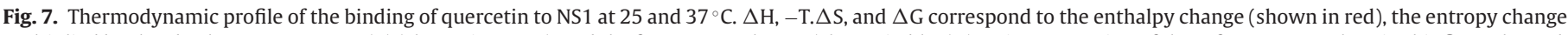

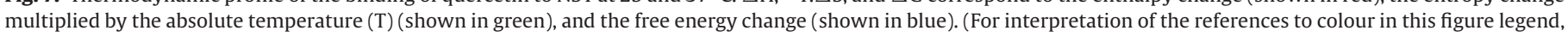
the reader is referred to the web version of this article.)

free energy $(\Delta G)$ and entropy changes $(\Delta S)$ for the NS1-quercetin complex were calculated from the following equations:

$\Delta G=-R T \ln \left(\frac{1}{K_{d}}\right)$

$\Delta S=\frac{(\Delta H-\Delta G)}{T}$

The thermodynamic parameters for the interaction between NS1 and quercetin are shown in Table 1 . The negative values obtained for the Gibbs free energy indicated that the interaction process is spontaneous. $\Delta H>0$ and $\Delta S>0$ suggest that the binding reaction is endothermic and enthalpically unfavorable, and furthermore, that hydrophobic interactions could play an important role in the formation of the NS1-quercetin complex [48-50]. The analysis of the enthalpy-entropy balance shows that the entropic term $(-T \Delta S)$ makes a favorable contribution to Gibbs free energy ( $\Delta G=\Delta H-T \Delta S$ ) (Fig. 7), suggesting that electrostatic interactions could be important in the stability of the NS1-quercetin complex [50]. Thus, the thermodynamic parameters indicated that the interaction between NS1 and quercetin presents both hydrophobic and electrostatic contributions.

\subsubsection{NS1 structure and its complex with quercetin}

The tridimensional structure of the protein NS1 has not been described in the literature. Therefore, a molecular model for the protein NS1 was predicted using ab initio structure prediction (Fig. 8). For the predicted model, a similar percentage of secondary structure elements ( $\alpha$-helix: $32 \%$ and $\beta$-sheet: $12 \%$ ) was found, compared with data obtained by circular dichroism ( $\alpha$-helix: $30 \%$ and $\beta$-sheet: $21 \%$ ). Furthermore, the similarity of secondary structures estimated by CD spectroscopy reinforces the NS1 modeling. An analysis of the model by the ProQ-SERVER resulted in a LG score of 2.302 and a Maxsub score of 0.011 [20]. Furthermore, the Ramachandran plot (Supplementary material, Fig. S2), calculated by PROCHECK [21], showed $90.2 \%$ of the residues lying in the most favorable regions and $9.8 \%$ in additional allowed regions. Therefore, the local and global geometric properties indicate a good model and validate the NS1 model.

The results of NS1 model MD simulation showed that the model is stable in solution, the RMSD value converged constantly, about $4 \AA$, from the model constructed by ROSETTA and the model clustered by GROMACS from the trajectories file, which retained the same structural characteristics (helix: $32 \%$; $\beta$-sheet: $12 \%$; turn and random coil: 56\%) (Supplementary material, Fig. S3).

Fig. 8 shows the docking result for the quercetin conformer of the lowest binding energy within the largest cluster. The binding energy between NS1 and quercetin for the docking result is $-29.7 \mathrm{~kJ} \mathrm{~mol}^{-1}$, which is in agreement with the values of Gibbs free energy present in Table 1. It can be observed from the hydrophobicity surface map in Fig. 8a that the interaction region of quercetin in NS1 presents a balance between hydrophilic and hydrophobic contributions. The theoretical distance determined between the single residue Trp of NS1 and quercetin was $20.12 \AA$ (Fig. 8b), which is in good agreement with the distance found by FRET analysis (19.06 $\AA$ ). The concordance present between the experimental and theoretical results for the binding energy and Trp-quercetin distance, reinforces our assertion that both protein and complex were modeled property.

Fig. 8c shows the analysis of interactions in the binding site of the NS1-quercetin complex performed by the LigPlot program [37]. His3, Tyr36, Asp70, Ile71, Cys72, Pro73, Ser105, and Leu143 residues. are involved in hydrophobic interactions with the flavonoid. His5, Ser69, Cys104, Gln106 and Asn108 residues form hydrogen bonds with the $\mathrm{O} 5, \mathrm{O} 7, \mathrm{O}^{\prime}, \mathrm{O} 3$, and 05 oxygen atoms of quercetin, respectively. The $\mathrm{NH}$ group of the His5 side chain makes up one hydrogen bond with the studied ligand with a formation distance of $2.86 \AA$ A. Each oxygen atom of Ser69, Cys 104, and the Gln106 main chain forms one hydrogen bond with the Quercetin, having 


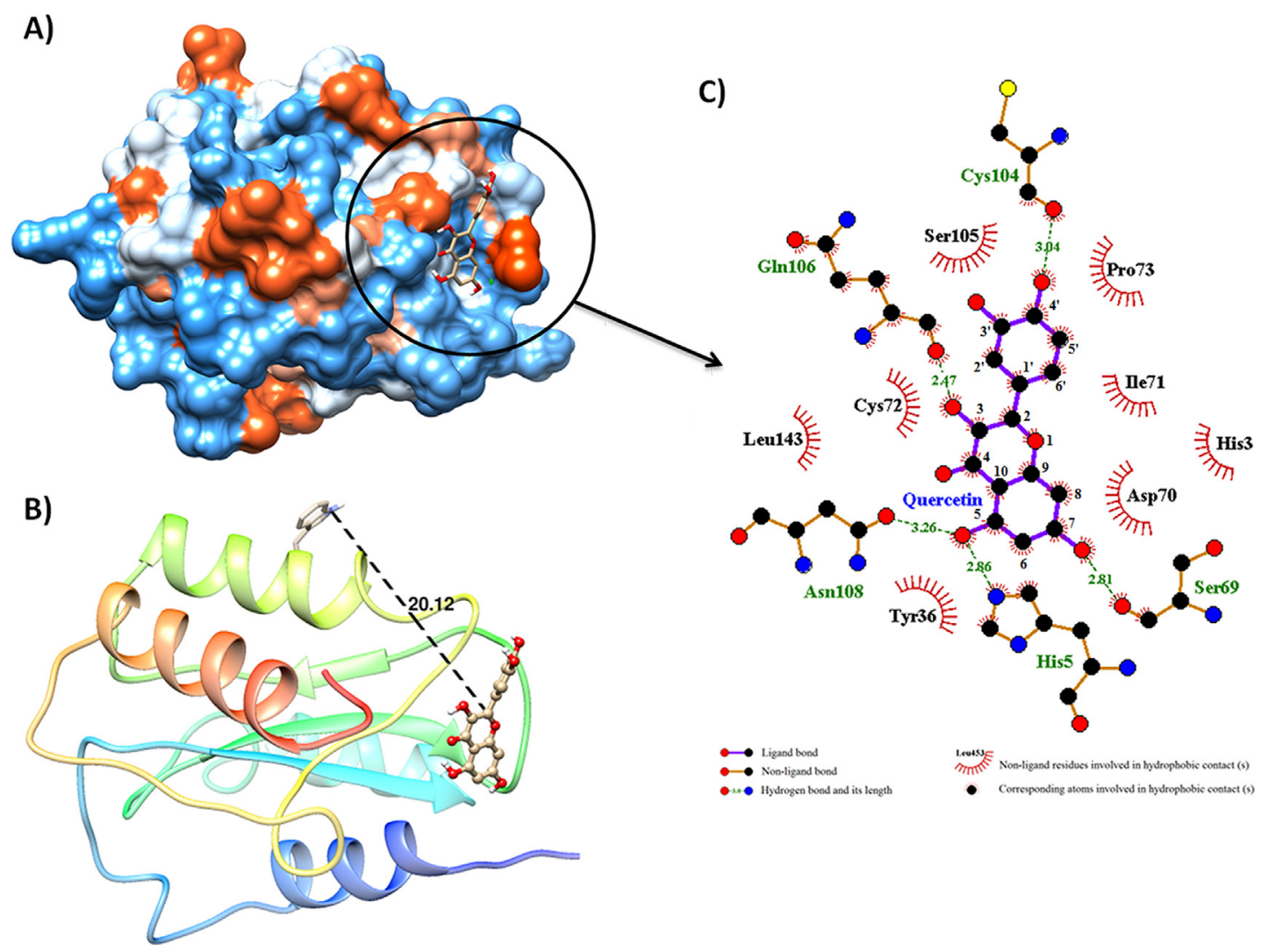

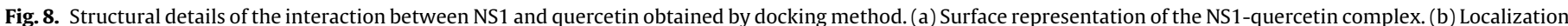

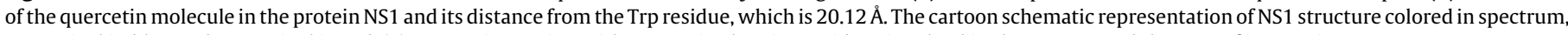
$n$-terminal in blue and $C$-terminal in red. (c) Interaction region with quercetin showing residues involved in the process and the type of interaction.

bond lengths of $2.81,3.04$, and $2.47 \AA$ Å, respectively. The side chain oxygen of the Asn108 residue builds up one hydrogen bond with the flavonoid with a formation distance of $3.26 \AA$.

Docking calculation analysis demonstrates that electrostatic interactions play an important role in the stability of the NS1quercetin complex, as well as hydrophobic contacts. These results corroborate the description of the enthalpy-entropy balance obtained from the thermodynamic analysis.

\section{Conclusions}

It is known that NS1 is involved in immune system subversion, inhibiting or neutralizing several steps in the interferon (IFN) pathway, as well as acting by silencing the ribonucleoproteic complex of hRSV. NS1 is able to decrease cellular levels of IKK $\varepsilon$ and TRAF3, which are components of the interferon cascade [4]. Furthermore, it interacts with many other cellular proteins, as described by Wu et al. [2]. Zandi et al. [8] tested the antiviral effects of flavonoids against type 2 Dengue Virus (DENV) in Vero cells; the results showed that quercetin exhibited significant anti-DENV replication properties, affecting intracellular DENV viral replication. In addition, Bachmetov et al. [9] have shown suppression of Hepatitis C Virus replication by quercetin, mediated by the inhibition of NS3 protease activity. Another study carried out by Choi et al. [10] showed the inhibition of viral replication in the initial stage of influenza A virus infection in the presence of quercetin 3rhamnoside. Finally, an antiviral activity assay using quercetin and other flavonoids to investigate plaque-forming unit (pfu) reduction [11] showed that quercetin presents infectivity and intracellular replication reduction properties against RSV. This scenario shows the importance of investigating interaction between viral proteins and flavonoids, in particular quercetin and its derivatives, principally regarding the description of the molecular mechanism in the interaction which leads to the prevention of viral infection.

The combination of experimental and in silico approaches showed that the interaction between hRSV-NS1 and quercetin is stable in the studied conditions, with a dissociation constant of the order of $10^{-6} \mathrm{M}$. The enthalpic and entropic energetic balance provided by thermodynamic analysis in addition to molecular modeling analysis indicates that the interactions involved in the formation and stabilization of the NS1-quercetin complex exhibits both hydrophobic and electrostatic contributions. From this perspective, the present results describe hRSV-NS1 as a potential target for future translational research.

\section{Author contributions}

Performed the experiments: Deriane Elias Gomes, Ícaro Putinhon Caruso and Isabella Otenio de Lourenço;

Performed theoretical studies: Gabriela Campos de Araujo and Ícaro Putinhon Caruso;

Analyzed the data: Deriane Elias Gomes, Ícaro Putinhon Caruso, Gabriela Campos de Araujo, Fernando Alves de Melo, Marinônio Lopes Cornélio, Marcelo Andrés Fossey, Fátima Pereira de Souza;

Wrote the paper: Deriane Elias Gomes, Ícaro Putinhon Caruso, Gabriela Campos de Araujo, Fernando Alves de Melo, Marinônio Lopes Cornélio, Marcelo Andrés Fossey, Fátima Pereira de Souza.

\section{Acknowledgments}

We thank Fundação de Amparo à Pesquisa do Estado de São Paulo - Fapesp (Grants 2010/50211-0 and 2010/52266-6) for finan- 
cial support and Prof. Dr. Márcio Colombo, Prof. Dr. Márcia C. Bisinoti and Prof. Dr. Altair B. Moreira for allowing the use of the spectrofluorimeter.

\section{Appendix A. Supplementary data}

Supplementary data associated with this article can be found, in the online version, at http://dx.doi.org/10.1016/j.ijbiomac.2015. 12.051 .

\section{References}

[1] Z. Ling, K.C. Tran, J.J. Arnold, M.N. Teng, Purification and characterization of recombinant human respiratory syncytial virus nonstructural protein NS1, Prot. Expr. Purific. 57 (2008) 261-270.

[2] W. Wu, K.C. Tran, M.N. Teng, K.J. Heesom, D.A. Matthews, J.N. Barr, J.A. Hiscoxa, The interactome of the human respiratory syncytial virus NS1 protein highlights multiple effects on host Cell Biology, J. Virol. 86 (2012) 7777-7789.

[3] S. Boyapalle, T. Wong, J. Garay, M. Teng, H. San Juan-Vergara, S. Mohapatra, S Mohapatra, Respiratory syncytial virus NS1 protein colocalizes with mitochondrial antiviral signaling protein MAVS following infection, PLoS One 7 (2012), e29386.

[4] S. Swedan, A. Musiyenko, S. Barik, Respiratory syncytial virus nonstructural proteins decrease multiple members of the cellular interferon pathways, J. Virol. 83 (19) (2009) 9682-9693.

[5] S. Swedan, J. Andrews, T. Majumdar, A. Musiyenko, S. Barik, Multiple functional domains and complexes of the two nonstructural proteins of human respiratory syncytial virus contribute to interferon suppression and cellular location, J. Virol. 85 (2011) 10090-10100.

[6] U. Hengst, P. Kiefer, Domains of human respiratory syncytial virus P protein essential for homodimerization and for binding to N and NS1 protein, Virus Genes 20 (2000) 221-225.

[7] B. Özçelik, I. Orhan, G. Toker, Antiviral and antimicrobial assessment of some selected flavonoids, Z. Naturforsch. C 61 (2006) 632-638.

[8] K. Zandi, B.T. Teoh, S.S. Sam, P.F. Wong, M.R. Mustafa, S. Abubakar, Antiviral activity of four types of bioflavonoid against dengue virus type-2, Virol. J. 8 (2011) 560 .

[9] L. Bachmetov, M. Gal-Tanamy, A. Shapira, M. Vorobeychik, T. Giterman-Galam, P. Sathiyamoorthy, A. Golan-Goldhirsh, I. Benhar, R. Tur-Kaspa, R. Zemel, Suppression of hepatitis $C$ virus by the flavonoid quercetin is mediated by inhibition of NS3 protease activity, J. Viral. Hepat. 19 (2012) e81-e88.

[10] H.J. Choi, J.H. Kim, C.H. Lee, Y.J. Ahn, J.H. Song, S.-H. Baek, D.H. Kwon, Antiviral activity of quercetin 7-rhamnoside against porcine epidemic diarrhea virus, Antiviral Res. 81 (2009) 77-81.

[11] T.N. Kaul, E. Middleton Jr, P.L. Ogra, Antiviral effect of flavonoids on human viruses, J. Med. Virol. 15 (1985) 71-79

[12] H.O. Gutzeit, Y. Henker, B. Kind, A. Franz, Specific interactions of quercetin and other flavonoids with target proteins are revealed by elicited fluorescence, Biochem. Biophys. Res. Commun. 318 (2004) 490-495.

[13] O. Gonzalez, V. Fontanes, S. Raychaudhuri, R. Loo, J. Loo, V. Arumugaswami, R Sun, A. Dasgupta, S.W. French, The heat shock protein inhibitor quercetin attenuates Hepatitis C virus production, Hepatology 50 (6) (2009) 1756-1764.

[14] M. Welch, A. Villalobos, C. Gustafsson, J. Minshull, You're one in a googol: optimizing genes for protein expression, J. R. Soc. Interface 6 (2009) S467-S476.

[15] E. Gasteiger, C. Hoogland, A. Gattiker, S. Duvaud, M.R. Wilkins, R.D. Appel, A. Bairoch, Protein Identification and Analysis Tools on the ExPASy Server, in: M. John Walker (Ed.), The Proteomics Protocols Handbook, Humana Press, 2005. pp. 571-607.

[16] N. Sreerama, R.W. Woody, A self-consistent method for the analysis of protein secondary structure from circular dichroism, Anal. Biochem. 282 (2000) $252-260$

[17] I.E. Borissevitch, More about the inner filter effect: corresctions of Stern-Volmer fluorescence quenching constants are necessary at very low optical absorption of the quencher, J. Lumin. 81 (1999) 219-224.

[18] A. Leaver-Fay, M. Tyka, S.M. Lewis, O.F. Lange, J. Thompson, R. Jacak, K. Kaufman, P.D. Renfrew, C.A. Smith, W. Sheffler, I.W. Davis, S. Cooper, A Treuille, D.J. Mandell, F. Richter, Y.E. Ban, S.J. Fleishman, J.E. Corn, D.E. Kim, S. Lyskov, M. Berrondo, S. Mentzer, Z. Popović, J.J. Havranek, J. Karanicolas, R. Das, J. Meiler, T. Kortemme, J.J. Gray, B. Kuhlman, D. Baker, P. Bradley, ROSETTA3: an object-oriented software suite for the simulation and design of macromolecules, Meth. Enzymol. 487 (2011) 545-574

[19] D. Shortle, K.T. Simons, D. Baker, Clustering of low-energy conformations near the native structures of small proteins, Proc Natl Acad Sci U. S. A. 95 (19) (1998) 11158-11162.

[20] B. Wallner, A. Elofsson, Can correct protein models be identified? Protein Sci. 12 (2003) 1073-1086.

[21] R.A. Laskowski, M.W. MacArthur, D.S. Moss, J.M. Thornton, PROCHECK-a program to check the stereochemical quality of protein structures, J. App. Cryst. 26 (1993) 283-291.

[22] L. Holm, P. Rosenström, Dali server: conservation mapping in 3D, Nucleic Acids Res. 38 (2010) W545-W549.
[23] H.J.C. Berendsen, J.P.M. Postma, W.F. Van Gunsteren, A. Dinola, J.R. Haak, Molecular dynamics with coupling to an external bath, J. Chem. Phys. 81 (8) (1984) 3684-3690

[24] S. Pronk, S. Páll, R. Schulz, P. Larsson, P. Bjelkmar, R. Apostolov, M.R. Shirts, J.C. Smith, P.M. Kasson, D. van der Spoel, B. Hess, E. Lindahl, GROMACS 4.5: high-throughput and highly parallel open source molecular simulation toolkit, Bioinformatics 29 (7) (2013) 845-854

[25] C. Oostenbrink, A. Villa, A.E. Mark, W.F. Van Gunsteren, A biomolecular force field based on the free enthalpy of hydration and solvation: the GROMOS force-field parameter sets 53A5 and 53A6, J. Comput. Chem. 25 (13) (2004) 1656-1676.

[26] H.J.C. Berendsen, J.P.M. Postma, W.F. Gunsteren, J. Van Hermans, Interaction Models for Water in Relation to Protein Hydration, in: B. Pullman (Ed.), Intermolecular Forces, 21, The Jerusalem Symposia on Quantum Chemistry and Biochemistry, 1981, pp. 331-342.

[27] G. Bussi, D. Donadio, M. Parrinello, Canonical sampling through velocity rescaling, J. Chem. Phys. 126 (1) (2007), 014101

[28] H.J.C. Berendsen, D. Van Der Spoel, R. Van Drunen, GROMACS: a message-passing parallel molecular dynamics implementation, Comput. Phys. Commun. 91 (1-3) (1995) 43-56.

[29] U. Essmann, L. Perera, M.L. Berkowitz, A smooth particle mesh Ewald method, J. Chem. Phys. 103 (19) (1995) 8577-8593.

[30] B. Rost, G. Yachdav, J. Liu, The PredictProtein server, Nucleic Acids Res. 32 (2004) 321-326

[31] X. Daura, K. Gademann, B. Jaun, D. Seebach, W.F. van Gunsteren, E. Mark, Peptide folding: when simulation meets experiment, Angew. Chem. Int. Ed. $38(1-2)(1999) 236-240$

[32] A.W. Schüttelkopf, D.M.F. van Aalten, PRODR: a tool for high-throughput crystallography of protein-ligand complexes, Acta Crystallogr. D 60 (2004) 1355-1363.

[33] M.F. Sanner, Python: a Programming Language for Software Integration and Development, J. Mol. Graph. Mod. 17 (1999) 57-61.

[34] M.H.M. Olsson, C.R. Søndergard, M. Rostkowski, J.H. Jensen, PROPKA3: consistent treatment of internal and surface residues in empirical pKa predictions, J. Chem. Theory Comput. 7 (2) (2011) 525-537.

[35] G.M. Morris, D.S. Goodsell, R.S. Halliday, R. Huey, W.E. Hart, R.K. Belew, A.] Olson, Automated docking using a larmarckian genetic algorithm and an empirical binding free energy function, J. Comput. Chem. 19 (1998) 1639-1662.

[36] E.F. Pettersen, T.D. Goddard, C.C. Huang, G.S. Couch, D.M. Greenblatt, E.C. Meng, T.E. Ferrin, UCSF Chimera -a visualization system for exploratory research and analysis, J. Comput. Chem. 25 (2004) 1605-1612.

[37] A.C. Wallace, R.A. Laskowski, J.M. Thomton, LIGPLOT: a program to generate schematic diagrams of protein-ligand interaction, Protein Eng. 8 (1995) $127-134$.

[38] E. Pretel, G. Camporeale, G. Prat-Gay, The Non-structural NS1 protein unique to respiratory syncytial vírus: a two-state folding monomer in quase-equilibrium with a stable spherical oligomer, PLOS ONE 8 (2013).

[39] J.M. Sanchez-Ruiz, J.L. López-Lacomba, M. Cortijo, P.L. Mateo, Differentia scanning calorimetry of the irreversible thermal denaturation of thermolysin, Biochemistry 27 (1988) 1648-1652.

[40] B. Sengupta, P.K. Sengupta, Binding of quercetin with human serum albumin: a critical spectroscopic study, Biopolymers (Biospectroscopy) 72 (2003) 427-434

[41] B. Mishra, A. Barik, K. Priyadarsini, H. Mohan, Fluorescence spectroscopy studies on binding of a flavonoid antioxidant quercetin to serum albumins, J. Chwm. Sci. 117 (2005) 641-647.

[42] O.J. Rolinski, A. Martin, D.J.S. Birch, Human serum albumin and quercetin interactions monitored by time-resolved fluorescence: evidence for enhanced discrete rotamer conformations, J. Biomed. Opt. 12 (3) (2007), 0340013.

[43] T. Förster, Intermolecular Energy Migration and Fluorescence, Ann. Phys. 2 (1948) 55-75.

[44] J.R. Lakowicz, Principles of Fluorescence Spectroscopy, 2nd ed., New York: Kluwer Academic Publishers/Plenum Press, 1999.

[45] J.R. Lakowicz, G. Weber, Quenching of fluorescence by oxygen. a probe for structural fluctuations in macromolecules, Biochemistry 12 (1973) 4161-4170.

[46] I. Matei, M. Hillebrand, Interaction of kaempferol with human serum albumin: a fluorescence and circular dichroism study, J. Pharm. Biomed. Anal. $51(2010) 768-773$

[47] D. Beckett, Measurement and analysis of equilibrium binding titration: a bignner's guide, Meth. Enzymol. 488 (2011) 1-16.

[48] I.P. Caruso, W. Vilegas, F.P. de Souza, M.A. Fossey, M.L. Cornelio, Binding of antioxidant flavone isovitexin to human serum albumin investigated by experimental and computational assays, J. Pharm. Biomed. Anal. 98 (2014) $100-106$

[49] I.P. Caruso, W. Vilegas, M.A. Fossey, M.L. Cornelio, Exploring the binding mechanism of guaijaverin to human serum albumin: fluorescence spectroscopy and computational approach, Spectrochim. Acta A Mol. and Biomol. Spectrosc. 97 (2012) 449-455.

[50] P.D. Ross, S. Subramanian, Thermodynamics of protein association reactions: forces contributing to stability, Biochemistry 20 (1981) 3096-3102. 